Feedback control of pl asma densi ty and heat ing power for st eady st at e operat i on i $n$ LHD

\begin{tabular}{|l|l|}
\hline $\begin{array}{l}\text { jour nal or } \\
\text { publ } \mathrm{i} \text { cat } \mathrm{i} \text { on } \mathrm{t} \text { i t l e }\end{array}$ & Fusi on Engi neer i ng and Desi gn \\
\hline vol une & 101 \\
\hline page range & $226-230$ \\
\hline year & $2015-10$ \\
\hline URL & ht t p: //hdl . handl e. net /10655/00012715 \\
\hline
\end{tabular}




\title{
Feedback Control of Plasma Density and Heating Power for Steady State Operation in LHD
}

\author{
Shuji Kamio ${ }^{a}$, Hiroshi Kasahara ${ }^{\mathrm{a}}$, Tetsuo Seki ${ }^{\mathrm{a}}$, Kenji Saito $^{\mathrm{a}}$, Ryosuke Seki ${ }^{\mathrm{a}}$, \\ Goro Nomura ${ }^{a}$, Takashi Mutoh ${ }^{a}$, and LHD Experiment Group ${ }^{a}$ \\ ${ }^{a}$ National Institute for Fusion Science, Toki 509-5292, Japan
}

\begin{abstract}
For steady state operation, the feedback control of plasma density and heating power system was developed in the Large Helical Device (LHD). In order to achieve a record of the long pulse discharge, stable plasma density and heating power are needed. This system contains the radio frequency (RF) heating power control, interlocks, gas fueling, automatic RF phase control, ion cyclotron range of frequency (ICRF) antenna position control, and graphical user interface (GUI). Using the density control system, the electron density was controlled to the target density and using the RF heating power control system, the RF power injection could be stable. As a result of using this system, we achieved the long pulse up to 48 minutes in the electron density of more than $1 \times 10^{19} \mathrm{~m}^{-3}$. Further, the ICRF hardware experienced no critical accidents during the 17th LHD experiment campaign in 2013.
\end{abstract}

Keywords: Steady state operation, Long pulse, Gas supply system, RF power control

\section{Introduction}

Steady state operation is important in fusion research, especially for revealing the physics related to the long pulse discharge, such as plasma wall interaction and divertor heat load. Research on the long pulse discharge has been demonstrated in many devices, using radio frequency (RF) heating power to sustain the plasma for a long period of time [1]. In the long pulse plasma discharge experiments in the Large Helical Device (LHD), ion cyclotron range of frequencies (ICRF) minority heating and electron cyclotron resonance heating (ECRH) are used for sustaining the plasma [2-4]. The minority heating is adopted in helium majority plasma and hydrogen minority plasma. Three pairs of antenna are used for the ICRF heating power injection, PA antenna [5], HAS antenna [4], and FAIT antenna [6]. These antennas each have transmitters and transmission lines, and each have loading resistance that depends on such conditions as plasma density and the antenna plasma gap. Improved long pulse discharges were difficult to attain because heating devices were delicate. In the previous experimental campaign, the principal reasons for plasma termination in the steady state operation were trouble on the heating devices or impurity contamination (i.e., insufficient power). However, for investigation of the steady state operation for fusion research, engineering problems must be solved.

To achieve the suitable long pulse discharge, the heating device power control system and the gas puffing control system are required. We upgraded the RF heating power control and gas puffing control system for steady state operation in the LHD experiments.

\section{The System Configuration}

Figure 1 shows the schematic view of the system configuration. The system works with the values of target density, RF power, and interlocks which input before or during the experiments. The system is made using a fast response hardware and software produced by National Instruments compact RIO (cRIO 9012 and its modules) and LabVIEW, respectively, to integrate the complicated feedback system. Using such instruments, the fast shutdown of the RF power by interlock could be mounted and intuitive operation was implemented using a graphical user interface (GUI). Table 1 shows the list of the channels of the system hardware for controlling the heating power, the oscillators, the antenna current phase, and the gas fueling. In order to operate feedback control, we have many input and output channels. The output signals for sustaining the LHD long pulse plasma are calculated from the input signals.

The system contains the RF heating power control, interlocks, gas fueling, automatic RF phase control, the ICRF antenna position control, and GUI. By employing the GUI system, the various kinds of experiment were demonstrated intuitively in the limited machine time on the LHD. During the steady-state plasma discharge, information such as plasma density, temperature, and injection power are displayed in real time. Therefore, researchers can respond immediately to the information received from the measured parameters. For the feedback control, the system contains the collection of the plasma measurement such as line averaged electron density $n_{e}$ measurement by the Far Infrared Rays (FIR) interferometer and neutral hydrogen emission $\mathrm{H}_{\alpha}$ and neutral helium emission He I. The values of the ICRF power transmission line, such as forward RF power $P_{f}$, reflection RF power $P_{r}$, peak voltage of the transmission line $V_{s}$, grid current on the oscillator $I_{g}$, and arcing sensor, also collected for the interlock system to inject stable RF power. The phase control system is also installed for changing the antenna current phase to the target value. In the long pulse discharge, we can change the current phase during a discharge and can investigate the ICRF heating 


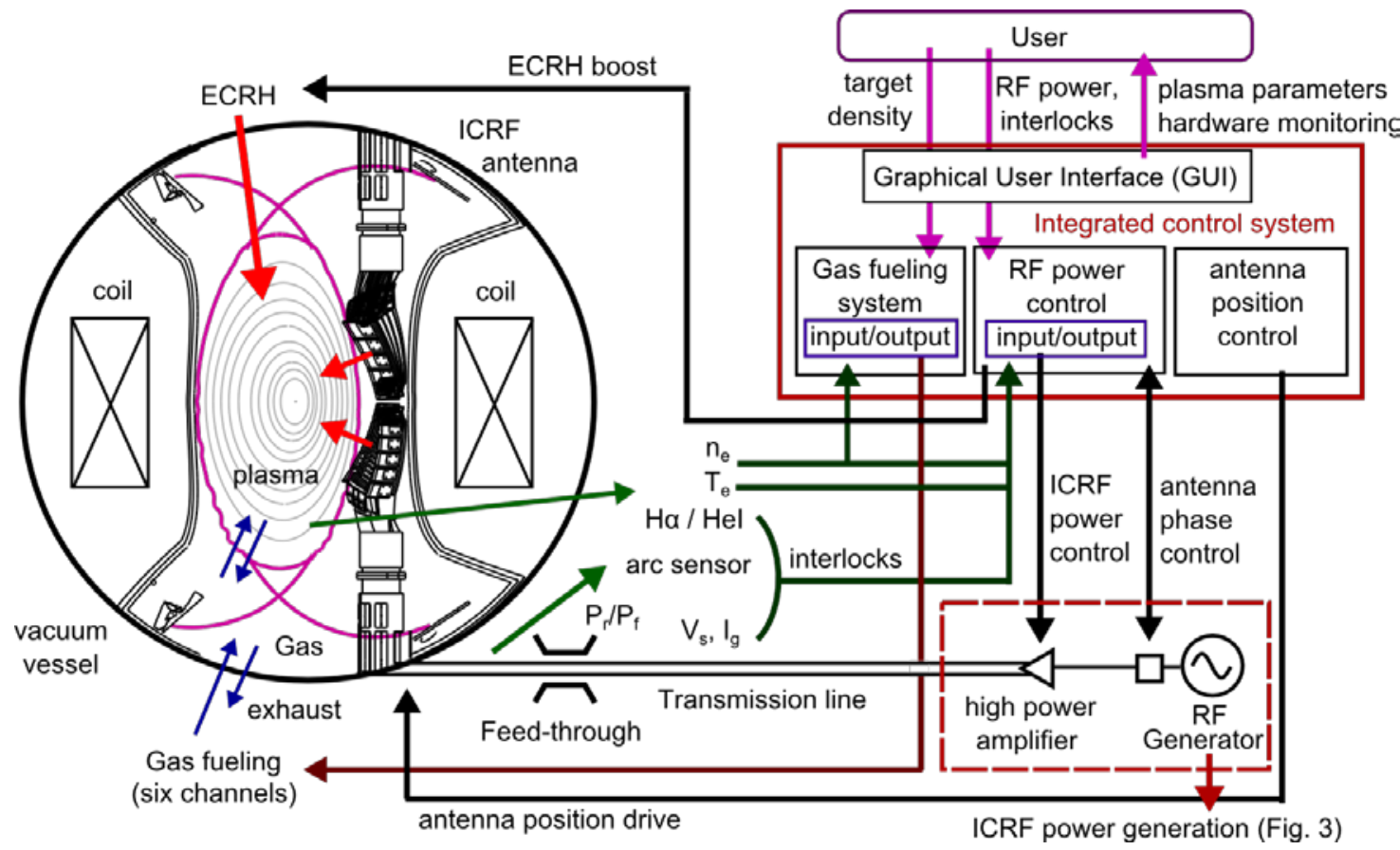

Fig. 1. Schematic view of the system configuration. This integrated control system contains the RF heating power control, interlocks, gas fueling, automatic RF phase control, the ICRF antenna position control, and GUI.

Table 1. Control channels of the NI hardware compact RIO 9012.

\begin{tabular}{|c|c|c|c|c|c|}
\hline Control & Signal & ch & Control & Signal & ch \\
\hline \multirow{3}{*}{$\begin{array}{c}\text { Heating } \\
\text { power }\end{array}$} & Input & 20 & \multirow{3}{*}{$\begin{array}{l}\text { Oscillato } \\
\text { rs for six } \\
\text { antennas }\end{array}$} & Input & 56 \\
\hline & & & & Output & 24 \\
\hline & TTLI/O & 64 & & TTLI/O & 48 \\
\hline \multirow{3}{*}{$\begin{array}{c}\text { Antenna } \\
\text { current } \\
\text { phase }\end{array}$} & Input & 20 & \multirow{3}{*}{$\begin{array}{c}\text { Gas } \\
\text { fueling }\end{array}$} & Input & 4 \\
\hline & Output & 16 & & Output & 16 \\
\hline & TTLI/O & 8 & & TTLI/O & 64 \\
\hline
\end{tabular}

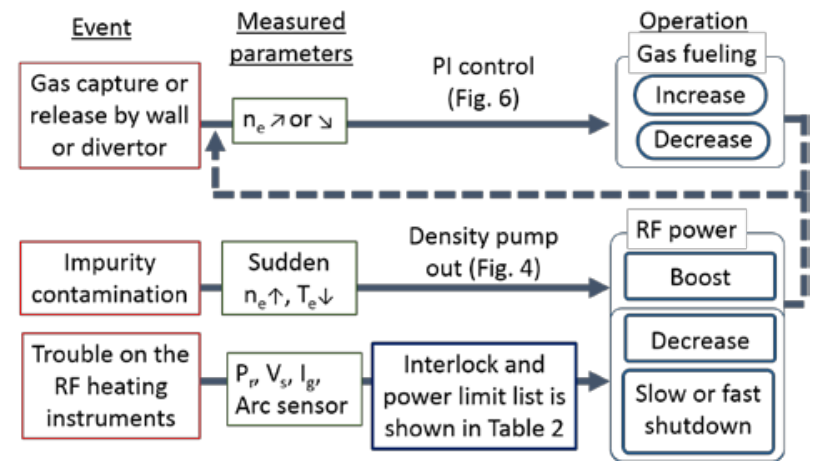

Fig. 2. Block diagram for the events and the operations.

characteristics with various antenna current phases. The RF power control system is explained in 2.1 and density control system is explained in 2.2. In this feedback control system, the RF heating power boost technique is employed for sustaining the plasma and decreasing the plasma density [7]. The operations of this system during various plasma events are summarized in Fig. 2 with a block diagram. The gas fueling rate and RF power are changed according to the plasma parameters, and vise vasa.

\subsection{RF Power Control System}

The ICRF power generation system is shown in Fig. 3 with the control system. Before the high power amplifier, we have two kinds of shutdown switches, power intensity adjustments, wave forming, and phase control. To achieve the suitable long pulse discharge, the first task is the restraint of the ICRF system for stable and safe operation. Stabilization of the ICRF injection power is important in sustaining the stable density of the plasma, because one of the limitations of the steady state operation in LHD before employing this system was termination of the ICRF injection power [3]. In order to stabilize, when an unexpected event such as increase of the ICRF power reflection occurs in the heating devices, immediate shutdown and restart are important aspects of the interlock system. In the developed system, many kinds of fast interlock systems are prepared for the safe operation, and their response times are set based on the oscillation of the plasma or the measurement noise because the interlock should not be triggered by the noise. Table 2 shows the ICRF power control systems with the typical threshold. The RF power must inject only into the plasma, hence the plasma density and emission must be confirmed. The ICRF power is controlled to be constant and not to increase the $V_{s}$ and $I_{g}$ by controlling the system gain. We control the ICRF forward power $\mathrm{P}_{\mathrm{f}}$ by the signal for the system gain. The interlock system has two time scales, slow and fast. The fast shutdown should be avoided because it may cause damage to the heating devices. For this reason, the slow shutdown is preferred. However, large $V_{s}, I_{g}$, and $P_{f}$ also cause damage to the heating devices or the transmission lines. Thus, in this particular case, the fast shutdown is more effective. When the slow interlock is triggered, the ICRF power gradually decreases 


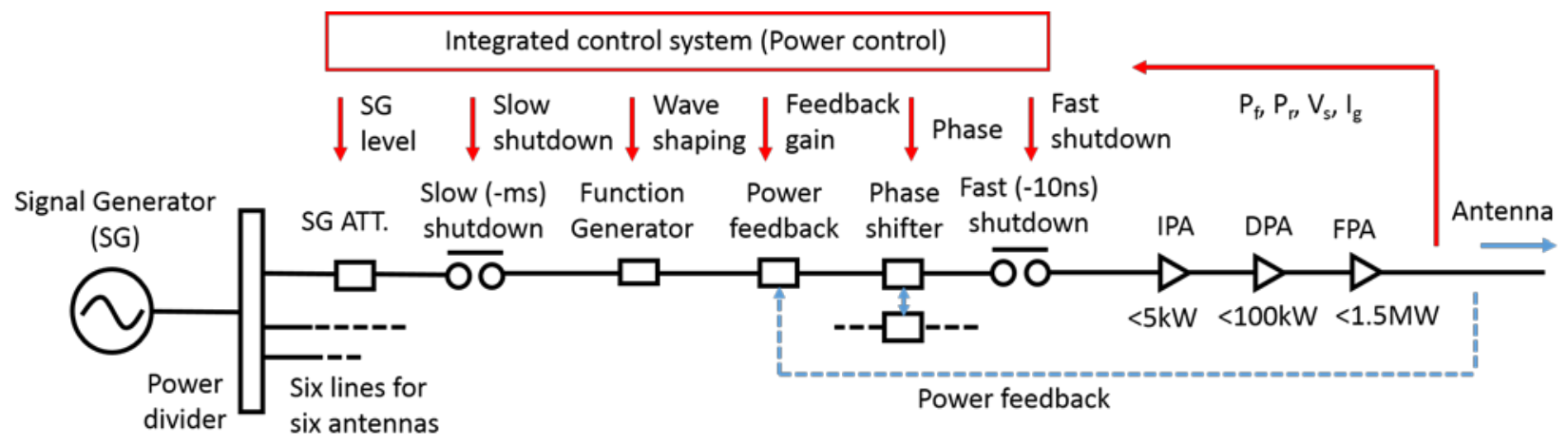

Fig. 3. Schematic view of the ICRF hardware and control system.

Table 2. ICRF Power Control Systems with Typical Threshold for the Hardware Safety Operation

\section{Plasma confirmation}

$n_{e}$

$$
>0.3 \times 10^{19} \mathrm{~m}^{-3}
$$

$\mathrm{H} \alpha$ or He I > noise level

\begin{tabular}{cll}
\hline \multicolumn{3}{c}{ ICRF power upper limit feedback } \\
$\mathrm{V}_{\mathrm{s}}$ & $<30 \mathrm{kV}$ & \\
$\mathrm{I}_{\mathrm{g}}$ & $<3 \mathrm{~A}$ & \\
\hline Interlocks & slow & fast \\
$\mathrm{Pr} / \mathrm{Pf}$ & $<0.2$ & \\
$\mathrm{Pr}$ & $<200 \mathrm{~kW}$ & \\
$\mathrm{Vs}$ & $<33 \mathrm{kV}$ & $<35 \mathrm{kV}$ \\
$\mathrm{Ig}$ & $<5 \mathrm{~A}$ & $<6 \mathrm{~A}$ \\
arc sensor & & $<$ noise level \\
\hline
\end{tabular}
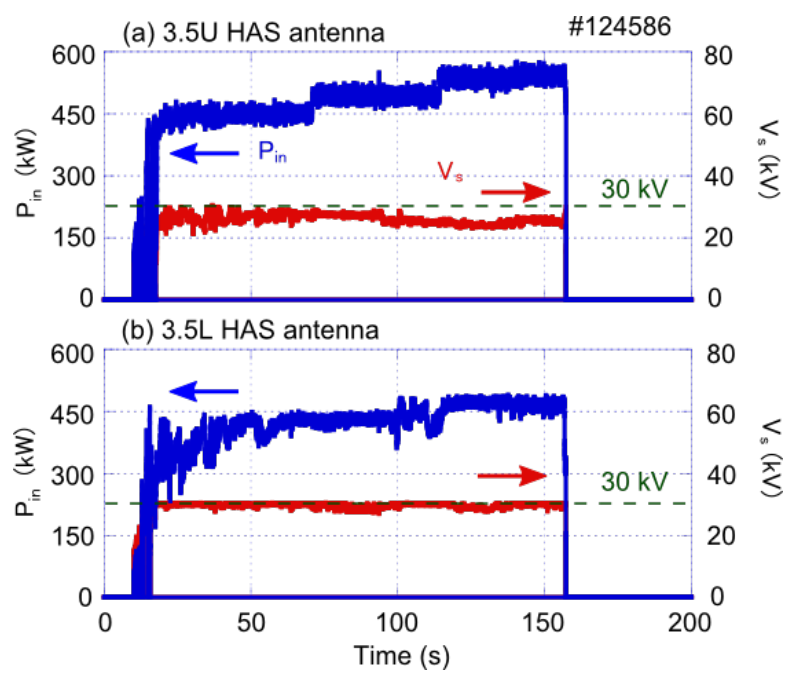

Fig. 4. Time evolution of the ICRF injection power $\mathrm{P}_{\text {in }}$ of the HAS antenna (a) 3.5U and (b) 3.5L, and peak voltage of the transmission line $\mathrm{V}_{\mathrm{s}}$. The 3.5L HAS antenna could not inject target power equal to the 3.5U antenna because the high voltage of the transmission line reached to $30 \mathrm{kV}$. for $25 \mu \mathrm{s}$ and then accelerates for $50 \mu \mathrm{s}$. When the fast interlock is triggered, the system shuts down the ICRF power in $100 \mathrm{~ns}$. The hardware of these interlocks is shown in Fig. 3. The interlock system engaged fine in the 17th cycle of the LHD experiment and there were no problems with the ICRF hardware.

The result of the power feedback by the upper limit by the $V_{s}$ is shown in Fig. 4. Figure 4 shows the time evolution of the ICRF injection power $\mathrm{P}_{\text {in }}$ and $\mathrm{V}_{\mathrm{s}}$ on the HAS antenna $U$ and $\mathrm{L}$. In this discharge, the 3.5L antenna could not inject target power equal to the 3.5U antenna because the high voltage of the transmission line reached to $30 \mathrm{kV}$. If the value of $\mathrm{V}_{\mathrm{s}}$ is larger than the upper limit, then the output voltage of the system gain is reduced by the feedback system. Employing this power limitation by $V_{s}$ and $I_{g}$, the arcing of the transmission line was reduced compared to the 16th cycle in 2012.

When a density increase event occurs due to the ionization of impurities or additional gas from the wall, we sustain the target density by decreasing the puffing gas and increasing the ICRF and ECRH injection power automatically by the system. Figure 5 shows the time evolution of (a) the line averaged electron density and (b) the ICRF and ECRH power in the steady state operation. A density increasing event occurred at $521.5 \mathrm{~s}$ and then RF power was immediately increased for sustaining the plasma and for decreasing the plasma density by the system. An ECRH antenna for short pulse operation was used to sustain the plasma at the moment of the event, and then for increasing the ICRF power in order to decrease the plasma density. After the ICRF heating power boost, the power is decreased slowly because, as can be seen in Fig. 5 (a) and (b), the density is affected by the changing of the ICRF power. The ECRH boost system is prepared by using an additional gyrotron and an ECRH antenna for using short pulse, and the ICRF boost is prepared by the power increasing on each of the six antennas. Generally, the ICRF matching deviates from the plasma density or the injection power [8]. However, the ICRF power is maintained by the matching feedback system [9] even when the density is changing, as shown in Fig. 5. 


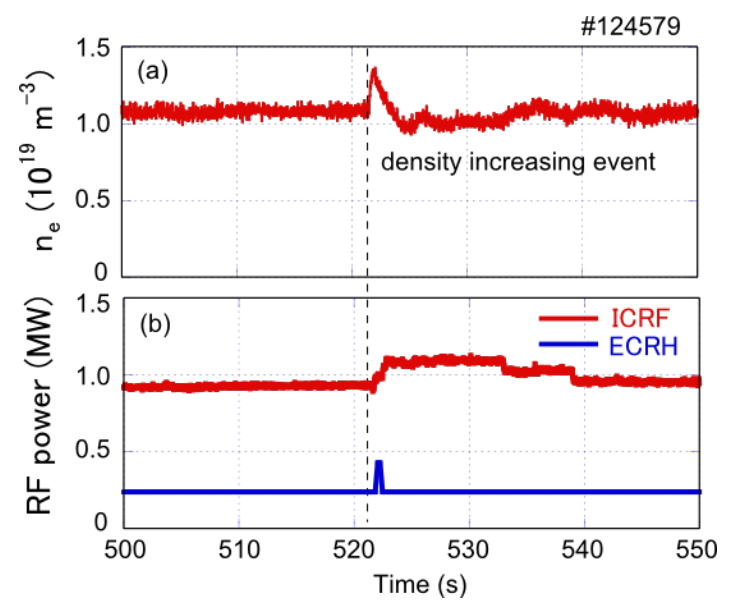

Fig. 5. Time evolution of (a) the line averaged electron density and (b) the ICRF and ECRH power in the steady state operation. After the density increasing event at $521.5 \mathrm{~s}$, the boost system triggered to sustain the plasma and decrease the plasma density.

\subsection{Plasma Density Control System}

The second task of this system is to sustain the plasma density and temperature by gas supply control. To increase the plasma density, the amount of the gas puffing needs to increase, and to decrease the plasma density, the amount of the gas puffing should decrease or inject higher RF power. The RF power boost technique in 2.1 is one of the density pump-out methods. Figure 6 shows the time evolution of the (a) RF power, (b) $n_{e}$ and target density, and (c) controlled supply voltage for opening the mass flow controller-s to puff the helium gas. The command density is changed during the discharge by an operator who is confirming the $\mathrm{n}_{\mathrm{e}}$ and the command density matching. Such operation is enabled by the GUI system. The system mainly controls the three sources of the gas supply. Mass flow controller-L for the initial large amount of the helium gas fueling, mass flow controller-s for control of the plasma density, and hydrogen fueling piezovalve for minority ratio control. As shown in Fig. 6, in the constant RF power injected discharge, the plasma density $\mathrm{n}_{\mathrm{e}}$ was well controlled by this system to the target density as shown in Fig. 6 (b). In this discharge, the ICRF matching control system was also automatically utilized even when the plasma density increased two or three times. The supplied voltage to the mass flow meter $\mathrm{V}_{\mathrm{MF}}$ is shown in Fig. 6 (c).

The gas supply is controlled based on the Proportional Integral (PI) of the measured $n_{e}$, because of the delay time shown in Fig. 7 (a)-(c). Figure 7 shows the time evolution of the (a) $n_{e}$, (b) supply voltage for the mass flow controller (s), and (c) supplied helium gas measured by the mass flow meter. The delay time from the $V_{\mathrm{MF}}$ to the helium puff is approximately $0.5 \mathrm{~s}$ and from the helium puff to the $\mathrm{n}_{\mathrm{e}}$ is approximately $0.3 \mathrm{~s}$.

$$
V_{\mathrm{MF}}=a\left(V_{0}-V_{n_{e}}\right)+V_{\text {slow }},
$$

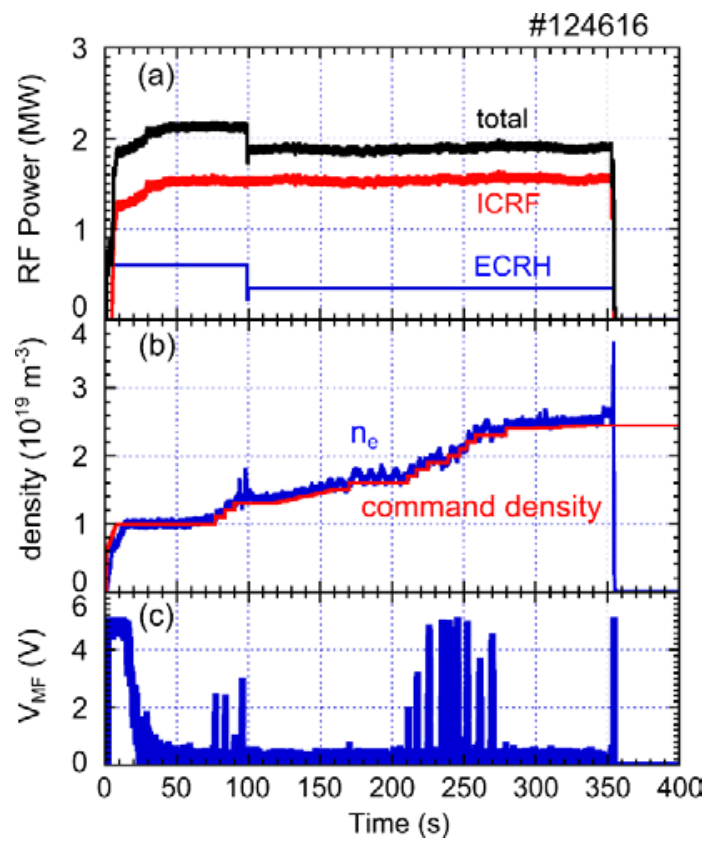

Fig. 6. Time evolution of the (a) RF power, (b) line averaged electron density ne and target density, and (c) controlled supply voltage for opening the mass flow controller (s) to puff the helium gas.

$$
V_{\text {slow }}(t+\mathrm{d} t)=V_{\mathrm{MF}}(t)+\frac{b}{\Delta t} \int_{t-\Delta t}^{t}\left(V_{0}-V_{n_{e}}\right) \mathrm{d} t,
$$

here $a$ is a fast reaction coefficient, $b$ is a slow reaction coefficient, $V_{\text {ne }}$ is a FIR output voltage, and $V_{0}$ is an FIR voltage corresponding to the target density. $a, b$, and $\Delta t$ were determined in the experiments. Even using the PI control, small density vibration appeared due to the delay times. This small vibration can be reduced by using the faster response gas puff mechanics, such as a piezo-valve, by designing a better controller with lower gains, or by taking the gas valve delay into account (e.g. Smith predictor).

\section{Use of Control to Achieve $\mathbf{4 8}$ min Discharge}

Using the system, we achieved the stable operation up to 48 minutes with electron density of more than $1 \times 10^{19} \mathrm{~m}^{-3}$. In the previous results on LHD before the system upgrade, the longest pulse length was 19 min because of the unstable RF power and uncontrollable plasma density [4]. The time evolution of the RF power, electron density $n_{e}$, and refueled quantity of helium gas in the achieved longest pulse discharge are shown in Fig. 8. In particular, the electron density $n_{e}$ was demonstrated as stable and constant, as shown in Fig. 8 (b). By achieving much longer pulse discharges than the other devices or achieved previously in LHD, many new research fields have emerged, such as wall particle inventory related to Fig. 8 (c) or divertor flakes contamination, which is the reason for the plasma termination of the discharge shown in Fig. 8. In this discharge, the RF heating power boost system was automatically triggered at the density increase events at $8 \mathrm{~min} 41 \mathrm{sec}$ and at $23 \mathrm{~min} 10 \mathrm{sec}$. 


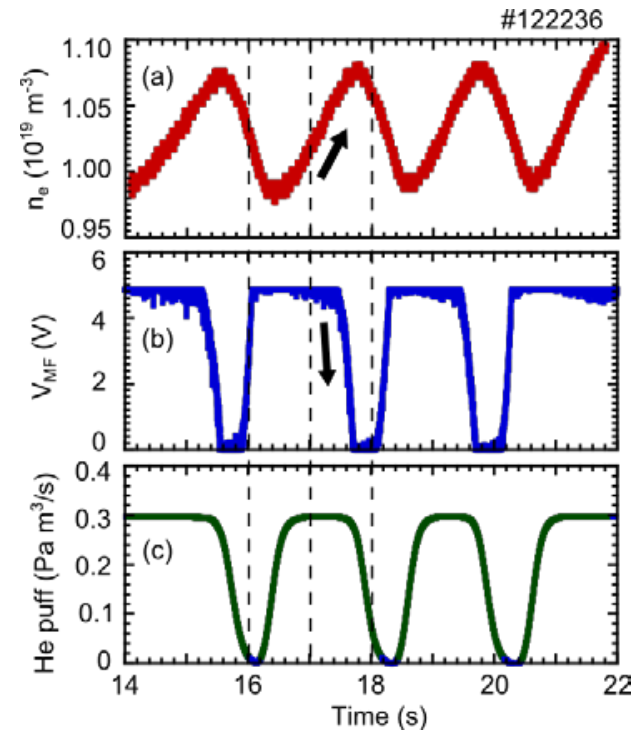

Fig. 7. Time evolution of the (a) line averaged electron density $n_{\mathrm{e}}$, (b) supply voltage for the mass flow controller (s), and (c) supplied helium gas.

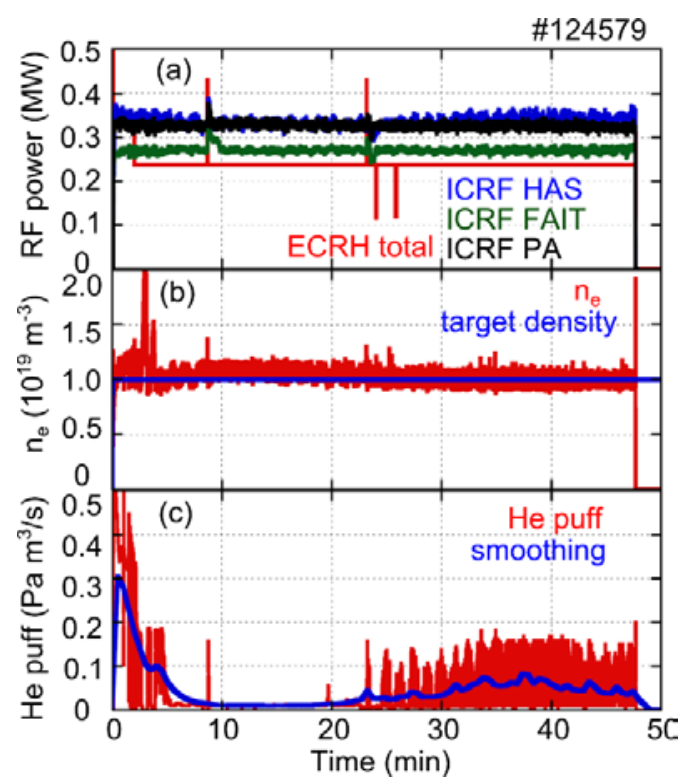

Fig. 8. Time evolution of the (a) RF power, (b) line averaged electron density $\mathrm{n}_{\mathrm{e}}$ and target density, and (c) refueled quantity of helium gas in the steady state operation achieved discharge up to 48 minutes.

\section{Summary and Conclusion}

The integrated RF power and plasma density control system was developed for steady state operation in the LHD experiments. The system contains the RF heating power control, interlocks, gas fueling, automatic phase control, antenna position control, and GUI. By the density control system, the electron density was controlled to the target density, and by the RF heating power control system the RF power injection could become stable. As a result of using this system, we achieved the long pulse up to 48 minutes in the electron density of more than $1 \times 10^{19}$ $\mathrm{m}^{-3}$, and no critical accidents with the ICRF hardware occurred in the 17th LHD experiment campaign in 2013. From this campaign, the steady state operation experiment has progressed to the next stage, where we will research plasma wall interaction, impurity behavior, heat flux to the divertor, and other issues related to problems in the heating devices. This system will extend control of the stub turner for matching the ICRF power and pellet injection for steady state operation in the 2015 experimental campaign.

\section{Acknowledgments}

This work was supported by NIFS Grant No. ULRR804.

\section{References}

[1] B. Saoutic et al., Tore Supra: Toward steady state in a superconduction tokamak, Fusion Sci. and Tech 56, 10791091 (2009).

[2] T. Mutoh et al., Steady-state operation and high energy particle production of $\mathrm{MeV}$ energy in the Large Helical Device, Nucl. Fusion 47, 9, 1250 (2007).

[3] Y. Yoshimura et al., Achievement of One Hour Discharge with ECH on LHD, J. Phys.: Conf. Ser. 25, 189-197 (2005).

[4] H. Kasahara et al., Development of steady-state operation using ion cyclotron heating in the LHD, Phys. Plasmas 21, 061505 (2014).

[5] T. Mutoh et al., Development and Fabrication of Steady State Fast Wave Antenna for LHD, J. Plasma Fusion Res. SERIES 1, 334-337 (1998).

[6] K. Saito et al., Field-aligned-impedance-transforming ICRF antenna in LHD, Fusion Eng. Des. (accepted).

[7] C. Angioni et al., Density response to central electron heating: theoretical investigations and experimental observations in ASDEX Upgrade, Nucl. Fusion 44, 827-845 (2004).

[8] Y. Lin et al., Digital real-time control for an ICRF fast ferrite tuning system on Alcator C-Mod, Fusion Eng. Des. 83, 2-3 (2008).

[9] K. Saito et al., Real-time impedance matching system for ICRF heating in LHD, Fusion Eng. Des. 83, 245-248 (2008). 